\title{
A three-year Strömgren photometric survey of suspected $\beta$ Pictoris-like stars ${ }^{\star}$
}

\author{
C. Nitschelm ${ }^{1}$, A. Lecavelier des Etangs ${ }^{1}$, A. Vidal-Madjar ${ }^{1}$, R. Ferlet ${ }^{1}$, E.H. Olsen ${ }^{2}$, and M. Dennefeld ${ }^{1}$ \\ 1 Institut d'Astrophysique de Paris, CNRS, 98 bis Bld. Arago, 75014 Paris, France \\ 2 Niels Bohr Institue for Astronomy, Physics and Geophysics, Astronomical Observatory, Juliane Maries Vej 30, DK-2100 \\ Copenhagen O, Denmark
}

Received January 4; accepted May 30, 2000

\begin{abstract}
We carried out a Strömgren photometric survey of thirteen southern bright stars, including $\beta$ Pictoris itself, during three years, d'Astrophysique de Paris, in order to detect possible weak photometric variations. $\beta$ Pictoris presents a small long-term variation with a change of brightness by $-2.110^{-3} \mathrm{mag}$ per year, over about two years from beginning of 1996, a situation relatively similar to the one about 18 years ago. Among the other stars, only HD 38392 presents weak photometric variations with a period of 21.4 days, probably related to the star rotation period. The negative result concerning photometric variations of all other stars suggests that those stars are actually really stable and strengthens the reality of the variations discovered in the case of $\beta$ Pictoris and HD 38392.
\end{abstract}

Key words: stars: $\beta$ Pic - circumstellar matter planetary systems - Strömgren photometry

\section{Introduction}

The main sequence stars with infrared excess are believed to be stars with dusty circumstellar disks (Aumann et al. 1984; Backman \& Paresce 1993; Vidal-Madjar et al. 1998). These so-called "debris-disks" must be a by-product of (young?) planetary systems activity like collisions between asteroidal objects (Backman \& Paresce 1993). Alternatively, the $\beta$ Pictoris disk could be the result of slow evaporation of Chiron-like objects (Lecavelier des Etangs et al. 1996). This disk has a very peculiar status in many ways: it has been extensively imaged in optical (see e.g. Mouillet et al. 1997) and thermal infrared (Lagage \& Pantin 1994). The spectroscopic surveys also

Send offprint requests to: C. Nitschelm

* Based on observations obtained at the Danish $50 \mathrm{~cm}$ telescope (SAT) at ESO, La Silla, Chile. revealed the presence of very fast spectroscopic variations interpreted by the passage in front of the star of comets in very eccentric orbits (Ferlet et al. 1987; Beust et al. 1991, 1998). In addition, the re-analysis of the photometric measurements done by the Geneva observatory until 1995 revealed that $\beta$ Pictoris also presents photometric variations (Lecavelier des Etangs et al. 1995a). Long term variations were observed from 1976 to 1981. Finally a large amplitude $(\gtrsim 0.04$ magnitude) and rapid variation was observed in November 1981 during about 10 days. This last variation has been interpreted by an occultation of the star by either a planet or a giant comet (Lecavelier des Etangs et al. 1995a,b, 1997; Lamers et al. 1997).

It thus appears of prime interest to make a photometric survey of $\beta$ Pictoris and similar main sequence stars. Such a survey should help to determine the frequency of occurrence of long-term variations among these stars. Moreover, rapid variations similar to the one of November 1981, which could be periodic, must be re-observed to discriminate between the different interpretations, and to find a definite explanation.

\section{The Strömgren photometric survey}

To compensate for the relatively small number of measurements obtained with our previous survey using the Geneva system between 1989 and 1997, a denser and long lasting photometric survey of $\beta$ Pictoris and of some other suspected $\beta$ Pictoris-like star candidates was needed. In October 1995 we began a circumstellar disk survey with the accurate Strömgren uvby photometric system being used almost permanently by the SAT group of the Copenhagen University Observatory. On behalf of a collaboration between Copenhagen Observatory and Institut d'Astrophysique de Paris through INSU (CNRS), we have performed three successive observational campaigns from 1995 to 1998, each season from October to April, using the SAT automatic $50 \mathrm{~cm}$ Danish telescope atop 
Table 1. List of the targets of the Strömgren uvby program. CMP means a comparison star for a Primary Target which reason to be selected in our program is given as follows: variable (VR), spectroscopic variable (SV), infrared excess (IR), with known circumstellar gas (CG). Campaign 1 refers to the austral summer 1995-1996, 2 to 1996-1997, 3 to 1997-1998. Data from CDS and the Bright Star Catalog (Hoffleit \& Warren 1991)

\begin{tabular}{|c|c|c|c|c|c|c|c|c|}
\hline & star & $*$ & $\alpha_{2000}$ & $\delta_{2000}$ & $m_{V}$ & Spectral type & $v \sin i$ & Campaigns \\
\hline \multirow{3}{*}{ HR 10} & HD 203 & CMP & $00 \mathrm{~h} 06 \mathrm{~m} 50.0 \mathrm{~s}$ & $-23^{\circ} 06^{\prime} 27^{\prime \prime}$ & 6.18 & F2IV & 155 & 3 \\
\hline & HD 256 & VR,SV,CG & $00 \mathrm{~h} 07 \mathrm{~m} 18.3 \mathrm{~s}$ & $-17^{\circ} 23^{\prime} 13^{\prime \prime}$ & 6.23 & $\mathrm{~A} 2 \mathrm{IV} / \mathrm{V}$ & 220 & $1,2,3$ \\
\hline & HD 693 & CMP & $00 \mathrm{~h} 11 \mathrm{~m} 15.8 \mathrm{~s}$ & $-15^{\circ} 28^{\prime} 04^{\prime \prime}$ & 4.89 & F5V & 8 & 2,3 \\
\hline \multirow{3}{*}{$\alpha$ Fornacis } & HD 16417 & CMP & $02 \mathrm{~h} 36 \mathrm{~m} 58.6 \mathrm{~s}$ & $-34^{\circ} 34^{\prime} 41^{\prime \prime}$ & 5.78 & G5IV & & 2,3 \\
\hline & HD 20010A & IR & $03 \mathrm{~h} 12 \mathrm{~m} 04.4 \mathrm{~s}$ & $-28^{\circ} 59^{\prime} 14^{\prime \prime}$ & 3.85 & $\mathrm{~F} 8 \mathrm{IV}$ & 0 & 2,3 \\
\hline & HD 17729 & CMP & $02 \mathrm{~h} 49 \mathrm{~m} 54.1 \mathrm{~s}$ & $-27^{\circ} 56^{\prime} 31^{\prime \prime}$ & 5.36 & $\mathrm{~A} 0 \mathrm{~V}$ & 135 & 2,3 \\
\hline \multirow{3}{*}{$\epsilon$ Eridani } & HD 21790 & $\mathrm{CMP}$ & $03 \mathrm{~h} 30 \mathrm{~m} 37.0 \mathrm{~s}$ & $-05^{\circ} 04^{\prime} 31^{\prime \prime}$ & 4.74 & B9Vs & 72 & 3 \\
\hline & HD 22049 & IR & $03 \mathrm{~h} 32 \mathrm{~m} 55.9 \mathrm{~s}$ & $-09^{\circ} 27^{\prime} 30^{\prime \prime}$ & 3.73 & $\mathrm{~K} 2 \mathrm{~V}$ & 8 & $1,2,3$ \\
\hline & HD 22243 & CMP & $03 \mathrm{~h} 34 \mathrm{~m} 37.4 \mathrm{~s}$ & $-09^{\circ} 52^{\prime} 05^{\prime \prime}$ & 6.25 & $\mathrm{~A} 2 \mathrm{~V}$ & 160 & $1,2,3$ \\
\hline \multirow{3}{*}{$o^{2}$ Eridani } & HD 22713 & $\mathrm{CMP}$ & $03 \mathrm{~h} 39 \mathrm{~m} \mathrm{01.1s}$ & $-05^{\circ} 37^{\prime} 34^{\prime \prime}$ & 5.96 & $\mathrm{~K} 1 \mathrm{~V}$ & & 3 \\
\hline & HD 26965A & VR & $04 \mathrm{~h} 15 \mathrm{~m} 16.3 \mathrm{~s}$ & $-07^{\circ} 39^{\prime} 09^{\prime \prime}$ & 4.41 & $\mathrm{~K} 1 \mathrm{~V}$ & & 2,3 \\
\hline & HD 25069 & CMP & $03 \mathrm{~h} 58 \mathrm{~m} 52.4 \mathrm{~s}$ & $-05^{\circ} 28^{\prime} 12^{\prime \prime}$ & 5.83 & G9V & & 3 \\
\hline \multirow{3}{*}{ HR 1307} & HD 26703 & CMP & $04 \mathrm{~h} 13 \mathrm{~m} 49.8 \mathrm{~s}$ & $+12^{\circ} 45^{\prime} 12^{\prime \prime}$ & 6.25 & K0 & & 3 \\
\hline & HD 26676 & IR & $04 \mathrm{~h} 13 \mathrm{~m} 34.5 \mathrm{~s}$ & $+10^{\circ} 12^{\prime} 45^{\prime \prime}$ & 6.23 & $\mathrm{~B} 8 \mathrm{Vn}$ & 300 & $1,2,3$ \\
\hline & HD 26793 & CMP & $04 \mathrm{~h} 14 \mathrm{~m} \mathrm{36.1 \textrm {s }}$ & $+10^{\circ} 00^{\prime} 41^{\prime \prime}$ & 5.22 & B9Vn & 350 & $1,2,3$ \\
\hline \multirow{3}{*}{$\zeta$ Dorado } & HD 32743 & CMP & $05 \mathrm{~h} 02 \mathrm{~m} 48.7 \mathrm{~s}$ & $-49^{\circ} 09^{\prime} 04^{\prime \prime}$ & 5.38 & $\mathrm{~F} 2 \mathrm{~V}$ & 0 & 3 \\
\hline & HD 33262 & IR & $05 \mathrm{~h} 05 \mathrm{~m} 30.6 \mathrm{~s}$ & $-57^{\circ} 28^{\prime} 21^{\prime \prime}$ & 4.72 & F7V & 0 & 2,3 \\
\hline & HD 35072 & $\mathrm{CMP}$ & $05 \mathrm{~h} 19 \mathrm{~m} \mathrm{22.1s}$ & $-50^{\circ} 36^{\prime} 21^{\prime \prime}$ & 5.45 & F7III-IV & & 3 \\
\hline \multirow{4}{*}{$\begin{array}{l}\gamma \text { Leporis B } \\
\gamma \text { Leporis A }\end{array}$} & HD 38382 & CMP & $05 \mathrm{~h} 44 \mathrm{~m} 28.3 \mathrm{~s}$ & $-20^{\circ} 07^{\prime} 35^{\prime \prime}$ & 6.34 & F8/G0V & & 3 \\
\hline & HD 38392B & VR & $05 \mathrm{~h} 44 \mathrm{~m} 26.5 \mathrm{~s}$ & $-22^{\circ} 25^{\prime} 18^{\prime \prime}$ & 6.15 & $\mathrm{~K} 2 \mathrm{~V}$ & & 2,3 \\
\hline & HD 38393A & IR & $05 \mathrm{~h} 44 \mathrm{~m} 27.7 \mathrm{~s}$ & $-22^{\circ} 26^{\prime} 27^{\prime \prime}$ & 3.60 & F7V & 15 & 2,3 \\
\hline & HD 35386 & CMP & $05 \mathrm{~h} 23 \mathrm{~m} 12.0 \mathrm{~s}$ & $-26^{\circ} 42^{\prime} 19^{\prime \prime}$ & 6.49 & F6V & & 3 \\
\hline$\zeta$ Leporis & HD 38678 & IR & $05 \mathrm{~h} 46 \mathrm{~m} 57.3 \mathrm{~s}$ & $-14^{\circ} 49^{\prime} 19^{\prime \prime}$ & 3.60 & A2Vann & 245 & 1,2 \\
\hline \multirow{3}{*}{$\delta$ Dorado } & HD 37935 & CMP & $05 \mathrm{~h} 36 \mathrm{~m} 55.1 \mathrm{~s}$ & $-66^{\circ} 33^{\prime} 36^{\prime \prime}$ & 6.30 & B9.5Ve & & 3 \\
\hline & HD 39014 & IR & $05 \mathrm{~h} 44 \mathrm{~m} 46.4 \mathrm{~s}$ & $-65^{\circ} 44^{\prime} 08^{\prime \prime}$ & 4.35 & $\mathrm{~A} 7 \mathrm{~V}$ & 206 & $1,2,3$ \\
\hline & HD 39963 & CMP & $05 \mathrm{~h} 51 \mathrm{~m} \mathrm{23.1 \textrm {s }}$ & $-64^{\circ} 02^{\prime} 01^{\prime \prime}$ & 6.36 & G8III & & 3 \\
\hline \multirow{3}{*}{$\beta$ Pictoris } & HD 35580 & CMP & $05 \mathrm{~h} 22 \mathrm{~m} 22.1 \mathrm{~s}$ & $-56^{\circ} 08^{\prime} 04^{\prime \prime}$ & 6.08 & $\mathrm{~B} 8.5 \mathrm{~V}$ & & $1,2,3$ \\
\hline & HD 39060 & VR,SV,IR,CG & $05 \mathrm{~h} 47 \mathrm{~m} 17.1 \mathrm{~s}$ & $-51^{\circ} 04^{\prime} 00^{\prime \prime}$ & 3.80 & A $5 \mathrm{~V}$ & 104 & $1,2,3$ \\
\hline & HD 40200 & CMP & $05 \mathrm{~h} 54 \mathrm{~m} 41.1 \mathrm{~s}$ & $-49^{\circ} 37^{\prime} 37^{\prime \prime}$ & 6.10 & B3V & & $1,2,3$ \\
\hline \multirow{3}{*}{ HR 2174} & HD 41692 & CMP & $06 \mathrm{~h} 06 \mathrm{~m} 38.7 \mathrm{~s}$ & $-04^{\circ} 11^{\prime} 38^{\prime \prime}$ & 5.39 & B5IV & 20 & 3 \\
\hline & $\mathrm{HD} 42111 \mathrm{~A}$ & CG & $06 \mathrm{~h} 08 \mathrm{~m} 57.8 \mathrm{~s}$ & $+02^{\circ} 29^{\prime} 58^{\prime \prime}$ & 5.73 & A3V & 120 & 3 \\
\hline & HD 42824 & CMP & $06 \mathrm{~h} 12 \mathrm{~m} 44.4 \mathrm{~s}$ & $-02^{\circ} 30^{\prime} 16^{\prime \prime}$ & 6.63 & $\mathrm{~A} 2 \mathrm{~V}$ & & 3 \\
\hline \multirow{3}{*}{51 Ophiuchi } & HD 155450 & CMP & $17 \mathrm{~h} 12 \mathrm{~m} 58.6 \mathrm{~s}$ & $-32^{\circ} 26^{\prime} 19^{\prime \prime}$ & 6.01 & B1II & 114 & \\
\hline & HD 158643 & $\mathrm{IR}, \mathrm{CG}$ & $17 \mathrm{~h} 31 \mathrm{~m} 24.8 \mathrm{~s}$ & $-23^{\circ} 57^{\prime} 46^{\prime \prime}$ & 4.81 & $\mathrm{~A} 0 \mathrm{~V}$ & 210 & 3 \\
\hline & HD 160915 & CMP & $17 \mathrm{~h} 43 \mathrm{~m} 25.7 \mathrm{~s}$ & $-21^{\circ} 41^{\prime} 00^{\prime \prime}$ & 4.87 & F6/F7V & 0 & 3 \\
\hline
\end{tabular}


La Silla Observatory (Florentin Nielsen et al. 1987; Florentin Nielsen 1993).

We report here the result of 3 years of observations of the stars listed in Table 1. This circumstellar program was conducted from 1995 to 1998 . As it could be noted only a small amount of stars were selected within our program, the reason being that only about a dozen of stars could be surveyed in a dense enough manner to be compatible with both the overall SAT observing program and the fact that at least two comparison stars should be observed simultaneously for each program star. The star list being extremely short, the program stars have to be selected in an extremely biased manner. They are in fact partly from our previous Geneva survey and partly as being "probably" good candidates, either because some variability is already suspected, or because they present some IR excess with known high $v \sin i$ value to favour the probability of extinction by dust within the disc.

Finally, during these three years as studies of dusty disks around A stars were made by several teams, some new stars were added to the program or suppressed from it during the chain of campaigns. Note that particularly $\beta$ Pictoris, HD 35580 (comparison, hereafter CMP), HD 40200 (CMP), HR 10, HD 22049, HD 22243 (CMP), HD 26676, HD 26793 (CMP) and HD 39014 were observed during all three years, whereas some other stars were observed during only one or two of our campaigns.

\section{Data analysis}

Using standard reduction programs, the uvby observations were reduced by the SAT group of the Copenhagen University Observatory (see Crawford \& Barnes 1970; Olsen 1994). The data are available in files containing the Henry Draper (HD) number of the star, the Julian Date (JD) of the observation, the air mass factor of the observation, the Strömgren $V$ magnitude, the $b-y$ color index, the $m_{1}=(v-b)-(b-y)$ and the $c_{1}=(u-v)-(v-b)$ parameters.

\subsection{Short term variations}

From our set of data, it was possible to search for short term as well as long term variations. The data analysis were performed on differences between stellar measurements and the corresponding measurements made on a comparison star. Short term variations would be detected if two subsequent measurements differ by more than 3 sigma from the average value of the data set. An eventual detection can be confirmed when comparing the star to the other comparison star. Over our three campaigns, no fluctuation was observed in any of our program stars except two of them, HR 10 and HD 38392 which will be discussed below.
This result already shows the quality of the survey since we obtained on the average the following precisions on the different parameters evaluated: $\sigma_{V} \lesssim 0.01 \mathrm{mag}$ for $V$ and $\sigma_{b-y} \lesssim 0.005 \mathrm{mag}$ for the $b-y$ color index. Within this accuracy, most of our surveyed stars did not present any short term variations, including $\beta$ Pictoris.

\subsection{Long term variations}

Concerning long term variations, we will illustrate here our approach directly in the case of $\beta$ Pictoris, since it is also the only case where some long range variation were detected.

Figure 1 shows the Strömgren $V$ magnitude evolution between October 1995 (JD 2450030) and March 1998 (JD 2450880) for $\beta$ Pictoris and its two comparison stars HD 35580 and HD 40200. It is clear that the three stars present similar variations which are thus not intrinsic variations but systematic residuals. However it is noteworthy that the statistical dispersion of individual data is at this stage of the order of only $\sigma \lesssim 0.008$ mag.

To detect possible weak variations, as in the case of our search for short term variations, we evaluate the difference between the data of a given star and its comparison star, provided that the measurements are contiguous in time. Practically, we set a maximum time difference between two measurements and we consider only the couples of measurements separated by less than 0.004 day ( $\sim 6 \mathrm{~min})$. This process is done for the Strömgren $V$ magnitude, the $b-y$ color index, the $m_{1}$ parameter and the $c_{1}$ parameter of all the stars. Figure 2 shows the magnitude difference between $\beta$ Pictoris and its two comparison stars (HD 35580 and HD 40200), whereas Fig. 3 shows the difference for the $b-y$ color index.

As already mentioned, no short-term variations are detected in the case of $\beta$ Pictoris. To detect long-term variations, we used the same method as described in Lecavelier et al. (1995a). We fitted the data of each star $(s)$ by a linear function of the time $(t):(a+b \cdot t)$ and evaluate the probability that the calculated slope $b_{\mathrm{s}}$ for the star $s$ is far from 0 assuming that $b=0$ is true. For a Gaussian distribution of the statistical noise in the data, the distribution of $b_{\mathrm{s}} / \sigma_{b_{\mathrm{s}}}$ must follow a Student law. Here we find that the Student law is well followed and consequently that the probabilities $P\left(b>b_{\mathrm{s}}\right)$ are uniformly distributed between 0 and 1 (see an example in Lecavelier et al. 1995a). For all the stars $(s)$ except $\beta$ Pictoris, we do not find a $b_{\mathrm{s}}$ value significantly different from 0 . This shows that our program stars as well as the comparison stars had no significant light variations during the survey. Again this underlines the quality of the survey and the strength of the very few real detections that are presented now. 
Table 2. List of the $U B V B_{1} B_{2} V_{1}$ G Geneva photometric system program stars. PT is for our main targets or "Primary Targets". CMP is for the comparison stars

\begin{tabular}{|c|c|c|c|c|c|c|c|}
\hline & star & $*$ & $\alpha_{2000}$ & $\delta_{2000}$ & $m_{V}$ & Spectral type & $v \sin i$ \\
\hline \multirow[t]{2}{*}{ HR 10} & HD 256 & $\overline{\mathrm{PT}}$ & $00 \mathrm{~h} 07 \mathrm{~m} 18.3 \mathrm{~s}$ & $-17^{\circ} 23^{\prime} 13^{\prime \prime}$ & 6.23 & $\mathrm{~A} 2 \mathrm{IV} / \mathrm{V}$ & 220 \\
\hline & HD 693 & CMP & $00 \mathrm{~h} 11 \mathrm{~m} 15.8 \mathrm{~s}$ & $-15^{\circ} 28^{\prime} 04^{\prime \prime}$ & 4.89 & F5V & 8 \\
\hline \multirow[t]{2}{*}{$\beta$ Pictoris } & HD 39060 & $\mathrm{PT}$ & $05 \mathrm{~h} 47 \mathrm{~m} 17.1 \mathrm{~s}$ & $-51^{\circ} 04^{\prime} 00^{\prime \prime}$ & 3.80 & $\mathrm{~A} 5 \mathrm{~V}$ & 104 \\
\hline & HD 35580 & CMP & $05 \mathrm{~h} 22 \mathrm{~m} 22.1 \mathrm{~s}$ & $-56^{\circ} 08^{\prime} 04^{\prime \prime}$ & 6.08 & $\mathrm{~B} 8.5 \mathrm{~V}$ & \\
\hline \multirow[t]{2}{*}{ HR 2174} & HD $42111 \mathrm{~A}$ & $\mathrm{PT}$ & $06 \mathrm{~h} 08 \mathrm{~m} 57.8 \mathrm{~s}$ & $+02^{\circ} 29^{\prime} 58^{\prime \prime}$ & 5.73 & $\mathrm{~A} 3 \mathrm{Vn}$ & 120 \\
\hline & HD 41692 & CMP & $06 \mathrm{~h} 06 \mathrm{~m} 38.7 \mathrm{~s}$ & $-04^{\circ} 11^{\prime} 38^{\prime \prime}$ & 5.39 & B5IV & 20 \\
\hline \multirow[t]{2}{*}{51 Ophiuchi } & HD 158643 & PT & $17 \mathrm{~h} 31 \mathrm{~m} 24.8 \mathrm{~s}$ & $-23^{\circ} 57^{\prime} 46^{\prime \prime}$ & 4.81 & $\mathrm{~A} 0 \mathrm{~V}$ & 210 \\
\hline & HD 155450 & CMP & $17 \mathrm{~h} 12 \mathrm{~m} 58.6 \mathrm{~s}$ & $-32^{\circ} 26^{\prime} 19^{\prime \prime}$ & 6.01 & B1II & \\
\hline \multirow[t]{2}{*}{ TY CrA } & $\mathrm{CD}-37^{\circ} 13024 \mathrm{~B}$ & $\mathrm{PT}$ & $19 \mathrm{~h} 01 \mathrm{~m} 40.1 \mathrm{~s}$ & $-36^{\circ} 52^{\prime} 33^{\prime \prime}$ & 9.54 & $\mathrm{Ae}$ & \\
\hline & HD 180885 & CMP & $19 \mathrm{~h} 19 \mathrm{~m} \mathrm{39.9s}$ & $-35^{\circ} 25^{\prime} 17^{\prime \prime}$ & 5.59 & B3V & 158 \\
\hline \multirow[t]{2}{*}{68 Ophiuchi } & HD 164577 & PT & $18 \mathrm{~h} 01 \mathrm{~m} 45.1 \mathrm{~s}$ & $+01^{\circ} 18^{\prime} 19^{\prime \prime}$ & 4.45 & $\mathrm{~A} 2 \mathrm{Vn}$ & 232 \\
\hline & HD 157089 & $\mathrm{CMP}$ & $17 \mathrm{~h} 21 \mathrm{~m} 07.0 \mathrm{~s}$ & $+01^{\circ} 26^{\prime} 35^{\prime \prime}$ & 6.95 & F9V & \\
\hline
\end{tabular}
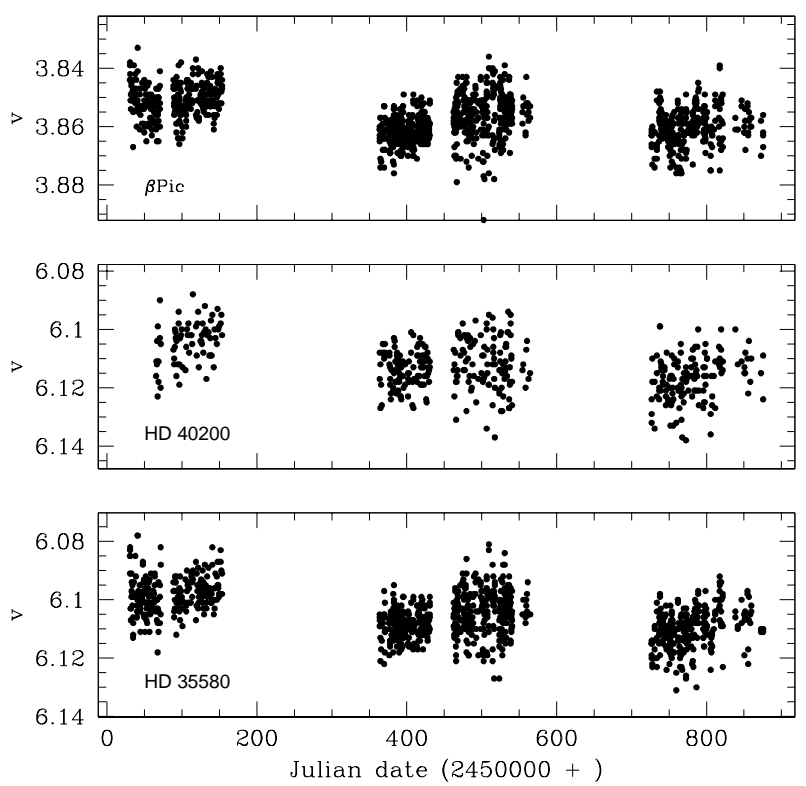

Fig. 1. Plot of the Strömgren $V$ magnitude of $\beta$ Pictoris, HD 40200 and HD 35580. We see that the data of the three stars present the same apparent signature of variations. These variations are obviously not real; they are only due to a residual noise identical for each star

\section{Results and discussion}

Will be presented and successively discussed in that section the three different types of variations detected during our survey.

\subsection{Comparison with the Geneva photometric survey}

In parallel to our main Strömgren program, the Geneva survey went on with the $70 \mathrm{~cm}$ Swiss telescope and the P7 $U B V B_{1} B_{2} V_{1} \mathrm{G}$ photometer at La Silla Observatory until its definitive end in 1997 (cf. Table 2). Figures 4 and 5 show the results for $\beta$ Pictoris and HR 10. Concerning $\beta$ Pictoris, apart from the variations discussed by Lecavelier
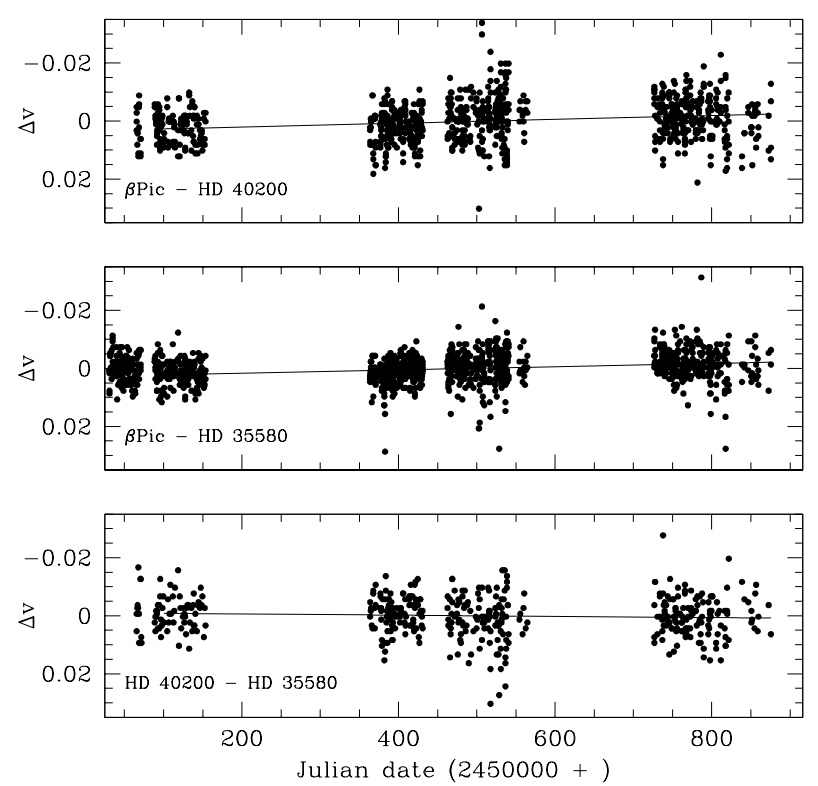

Fig. 2. Plot of the magnitude differences of the same three stars. Here it is clear that the apparent variations visible in the previous figure have been removed. The difference between the two comparison stars is flat, and neither short term nor long term variations are detected over the three years of observations (bottom panel). This is exactly the case of all other stars of the program. In contrast, $\beta$ Pictoris shows a long term variation with a significant slope which furthermore and as expected, does not depend upon the comparison star used for the subtraction

et al. (1995a), no other variations were significantly detected in these data, except a slight increase of the star brightness from 1995 (Fig. 4). This increase is marginal and at the limit of the detectability on this survey with a variation slope of $(-5.1 \pm 2.5(1 \sigma)) 10^{-6}$ mag per day. This is however noteworthy because this can be exactly superimposed on the variation detected with the Stömgren survey which will be discussed in Sect. 4.3. 

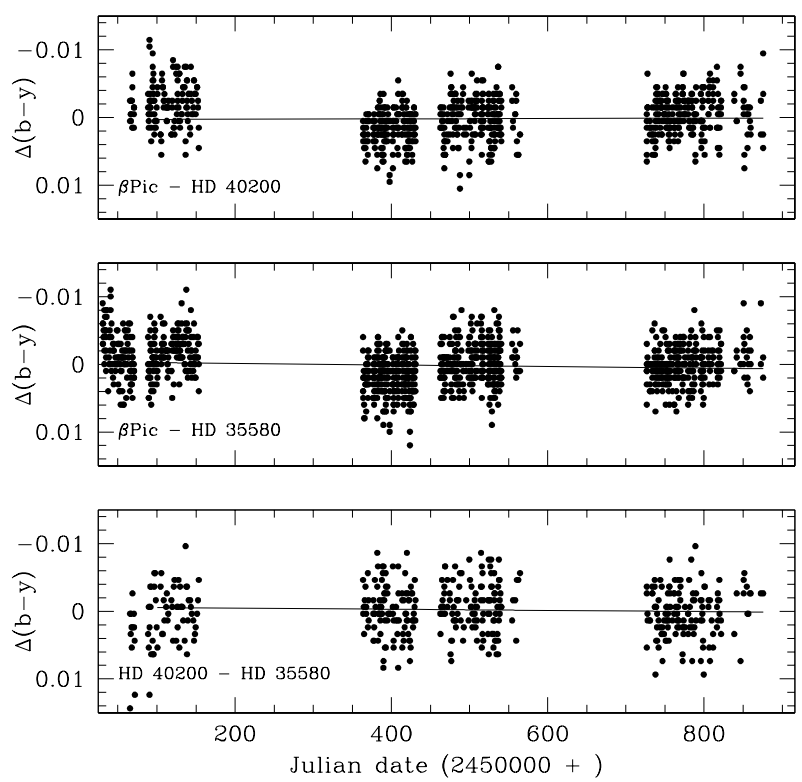

Fig. 3. Same as the previous figure but for the $b-y$ color index. The slopes of the three differences (solid lines) are not significantly different from zero. The long term variations observed in the case of $\beta$ Pictoris are thus relatively achromatic

For HR 10, we have already detected a short-term variation in January 1990 (Lecavelier et al. 1995a, and Fig. 5). The new data obtained since 1995 also show an apparent group of measurements around December 25, 1996 (Julian Date JD 2450443) during which the star appeared fainter than normally. But a careful look reveals that this group consists of three isolated measurements of lower quality, separated by good quality measurements at the normal brightness level (Fig. 5). We thus conclude that this apparent variation is not significant.

TY CrA is well known as an high amplitude eclipsing binary. We observed it to search for long term variations. Except one very short term light decrease related to an eclipse, no significant long term variations were observed.

68 Ophiuchi has been removed from the SAT program. This star was included in the Geneva program because of the suspected presence of circumstellar dust which was dismissed by subsequent observations (Vidal-Madjar et al. 1995; Waters et al. 1995).

\subsection{HD 38392 periodic variations}

We were surprised to see in our search for short term variations that one of our stars, HD 38392 was presenting a surprisingly high magnitude of fluctuation, its sigma being at least twice the one observed in the case of the other stars of our program. Because, as already said, its long term variation was not presenting any trend, we tried to find out the cause of such a behaviour.

The magnitude difference between the stars HD 38392 and HD 38393 is shown for illustration as a function of the

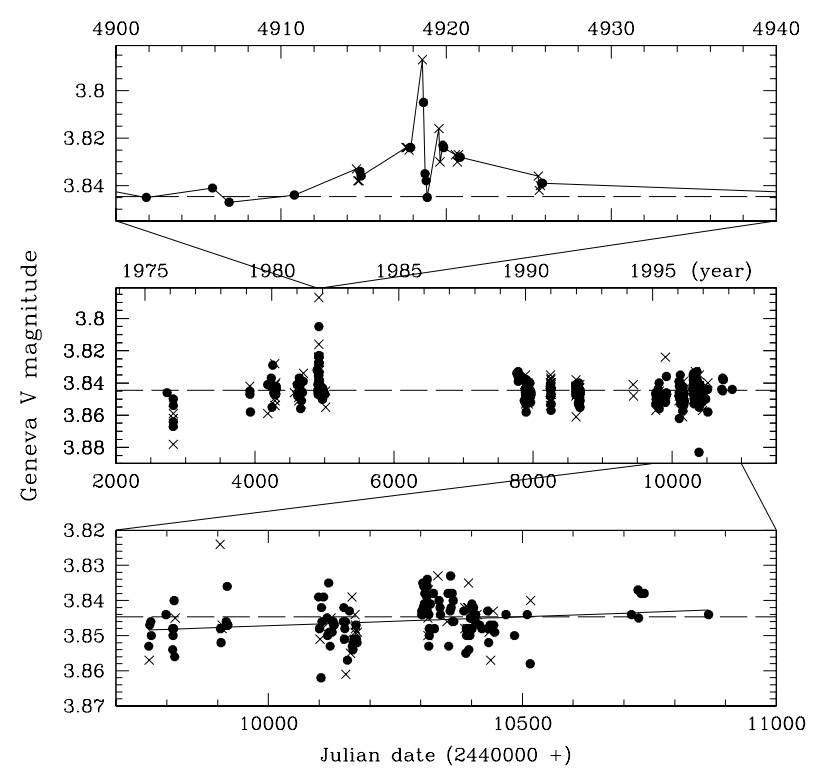

Fig. 4. Plot of the Geneva $V$ magnitude for $\beta$ Pictoris from 1976 to 1997 (middle panel). The filled circles represent the good quality data (weight $\geq 3$ ) and crosses represent the lower quality measurements (weight $<3$ ). This plot show the new data obtained from 1995 to 1997 in addition to the Fig. 1 of Lecavelier et al. (1995a). The upper panel show the rapid variation observed around November, 10 1981. The bottom panel show the long-term variation marginally detected here from 1995 and the corresponding linear fit to the data

time (Fig. 6, top panel). Differences with the two other comparison stars definitely show that variations are due to HD 38392 and not to HD 38393.

From the observation of these data it appears that a periodic variation is present in the data. This was searched for by standard Fourier analysis and a very clear period of 21.4 days showed up immediately. Folding the data in phase, it shows a clear stellar variation of about 0.02 magnitude (Fig. 6, bottom panel), compatible with a fluctuation due to stellar activity with a rotation period of the star of the order of 21 days.

Note that this period was not previously known but only indirectly inferred (e.g. Soderblom et al. 1991, infer 12.8 days; Stepień \& Geyer 1995, infer 20 days) from the correlation between the emission flux in the $h$ and $k$ lines of MgII and the rotation period. Here we directly observed the stellar rotation period if our interpretation is correct.

\section{3. $\beta$ Pictoris long term variations}

In the $\beta$ Pictoris case, the calculated slope $b_{\beta \text { Pic }}$ is significantly different from 0 . The probability to have a so large slope $b_{\beta \text { Pic }}$ only by chance and due to the statistical noise is $P\left(b>b_{\beta \text { Pic }}\right) \ll 10^{-7}$. The fits to the $\beta$ Pictoris data (solid lines in Figs. 2 and 3) start from JD 2450100 because before this date, data were obtained only with HD 35580 as comparison star. 


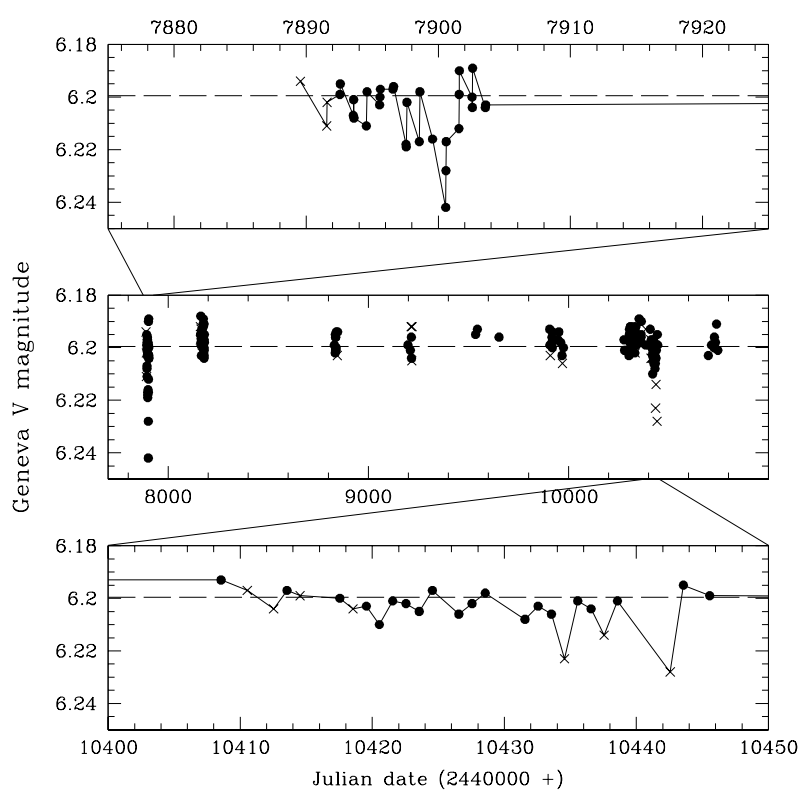

Fig. 5. Plot of the Geneva $V$ magnitude measured on HR 10 from 1990 to 1997 (middle panel). The two other panels show the times when the star appeared fainter than normal in January 1990 (top panel) and December 1996 (bottom panel). The filled circles represent the good quality data (weight $\geq 3$ ) and crosses represent the lower quality measurements (weight $<3$ ). It is clear that the variation in 1990 was real (and observed in other bands not shown here) while the apparent variation in late 1996 is only due to 3 lower quality measurements separated by good quality measurements at normal brightness

The fit gives a slope $(-6.55 \pm 1.18(1 \sigma)) 10^{-6} \mathrm{mag}$ per day if we use HD 40200 as comparison star, and a slope $(-5.56 \pm 0.75(1 \sigma)) 10^{-6} \mathrm{mag}$ per day with HD 35580 as comparison star. Assuming that the distributions are Gaussian, we can combine the two slopes and we find that the variation of the $\beta$ Pictoris magnitude was $(-5.84 \pm$ $0.64(1 \sigma)) 10^{-6} \mathrm{mag}$ per day. This corresponds to a variation of $-2.110^{-3} \mathrm{mag}$ per year.

As a conclusion, the brightness of $\beta$ Pictoris significantly increased from JD 2450100 (January 17, 1996) to JD 2450876 (March 3, 1998) by about $4.510^{-3}$ magnitude.

\section{Constraints on future $\beta$ Pictoris observations}

In the $\beta$ Pictoris observations, no rapid light variations, neither in $V$ magnitude, in the $b-y$ color index nor in the $m_{1}$ and $c_{1}$ parameters were detectable during the three campaigns (1995-1996, 1996-1997 and 1997-1998). This is nevertheless interesting and significant. It is now clear that an event like the one, which occurred in November 10, 1981, is very rare. Assuming that the event of November 1981 is periodic, and using the available data with the Geneva and Strömgren observations, it is now possible to eliminate a large range of value for the putative period. Each given measurement at time $t$ which does not show
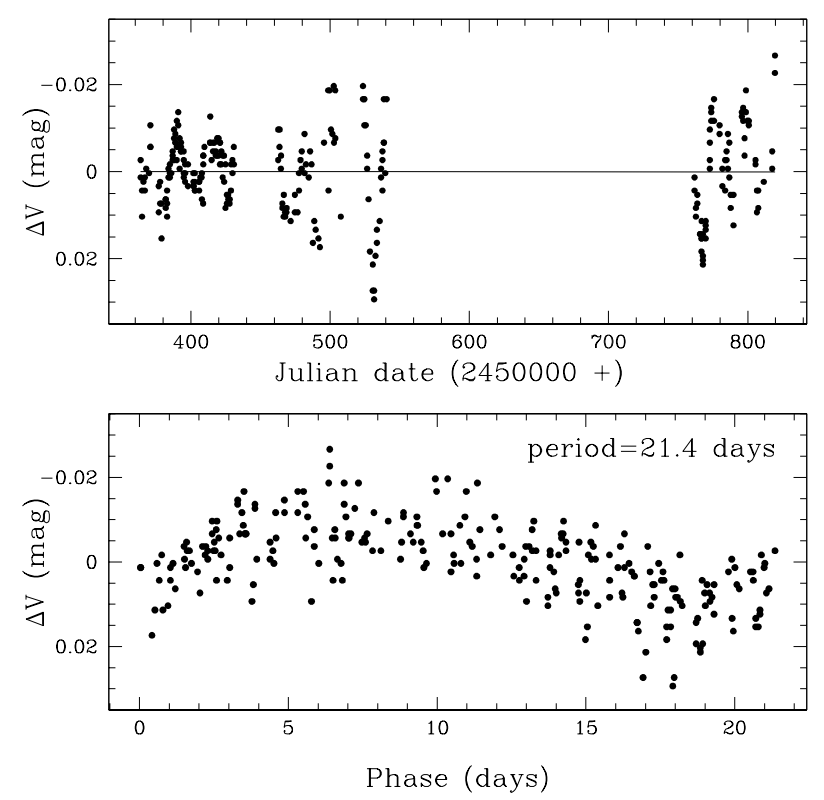

Fig. 6. Top panel presents the magnitude difference between HD 38392 and HD 38393 from the data gathered during three campaigns between 1995 and 1998. The slope of the linear fit to the data is very close to zero (solid line, top panel). There is no long-term variation for these two stars. Bottom panel shows the periodic variation detected for HD 38392 with a period of 21.4 days corresponding to its rotational period (Stepień \& Geyer 1995)

this variation allows to eliminate the period $P_{1}$ given by the time difference between the measurement and the date November 10: $P \neq P_{1}=t-t_{11 / 10 / 1981}$.

In fact, considering that if this event had occurred again, it would have been detectable during one day (which is a very conservative assumption), each measurement allows to eliminate the range of period $P_{1}$ plus or minus half a day. Moreover, each period $P_{n}$ resulting from the division of $P_{1}$ by an integer $n$ can also be eliminated. In other words, for each measurement at time $t$, the ranges of period $\left[\left(P_{1}-0.5\right) / n ;\left(P_{1}+0.5\right) / n\right]$, where $n$ is an integer, are not possible. The resulting set of impossible periods is plotted in Fig. 7 for the Geneva and Strömgren data. From this plot we see that $90 \%$ of periods between 0 and 1000 days can be eliminated. $49 \%$ of periods between 1000 and 2000 days, $29 \%$ of periods between 2000 and 3000 days and $57 \%$ of periods between 5000 and 6000 days are also eliminated. To cover the periods between 3000 and 5000 days, new observations are required from nowadays to the year 2009 .

\section{Conclusion}

We performed a photometric survey of the star $\beta$ Pictoris and a few $\beta$ Pictoris-like candidates during a total of about eighteen months from 1995 to 1998, using the automatic $50 \mathrm{~cm}$ Danish telescope atop La Silla Observatory and 


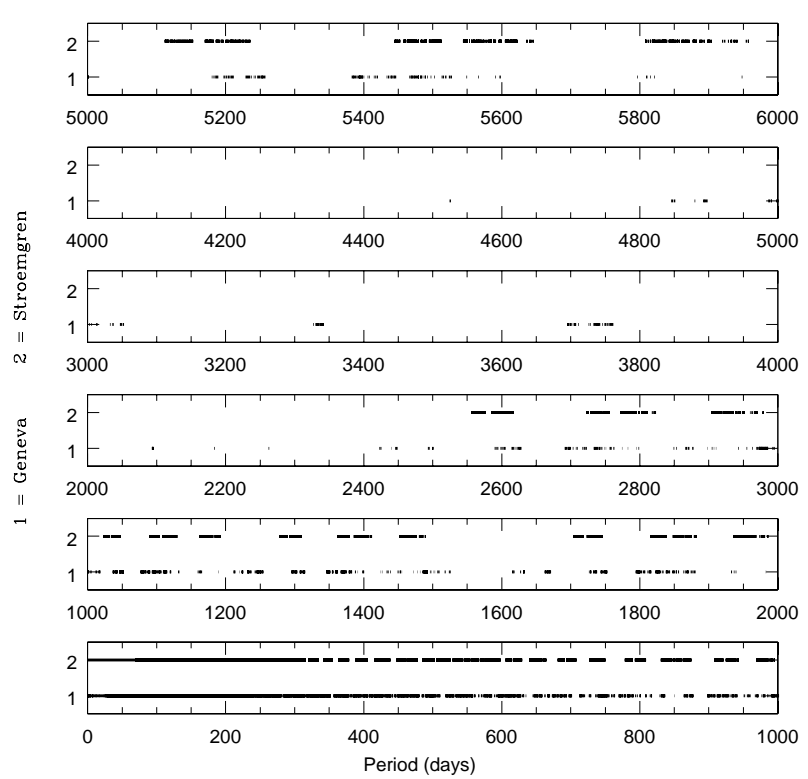

Fig. 7. Plot of the impossible periods for the event of November 10, 1981 calculated from the data of the Geneva observatory (1) and the Strömgren system (2). Between 0 and 1000 days, $90 \%$ of the periods are impossible. Periods between 5000 and 6000 days are eliminated by the non-observations of rapid variations in the data collected recently from 1995. Whole fractions of these periods are also eliminated and allow to conclude that about $50 \%$ of the periods between 1000 and 2000 days and between 2500 and 3000 days are not possible

its Strömgren uvby photometer. No star observed during this campaign except HD 38392 and $\beta$ Pictoris showed any significant variations. This negative result reveals the quality of the observations and enforces the reality of the two variations observed. This also seems to indicate that short and long-term variations for these $\beta$ Pictoris-like stars must be exceptional.

In the case of HD 38392, a periodic variation was clearly detected with a period of 21.4 days, probably related to the stellar rotation period. Having some activity at its surface, this induces some weak photometric variations in all indexes followed in our survey.

Concerning $\beta$ Pictoris, a new long-term variation has been seen with an increase of the star brightness starting from January 1996. These long-term light variations, which could be due to inhomogeneous distribution of the dust within the disks, are very weak and need several years of observation to be detected. It is interesting to recall that the photometric event in November 1981 was observed after such a slow increase of the star brightness in the preceding years (Lecavelier et al. 1995a). It thus appears important to continue the photometric survey of this star with a time coverage as dense as possible.

Acknowledgements. We are grateful to the Copenhagen University Observatory SAT group, led by Jens Viggo Clausen, which supplied preliminary reductions on the daily raw data. We are also very grateful to the observers who spent a lot of time at La Silla Observatory to ensure the successful execution of the SAT program, i.e. J.-P. Beaulieu, Jens Viggo Clausen, Rosa Clément, Frédéric Derue, Bodil E. Helt, Helge Jonch-Sorensen, Bjarne R. Jorgensen, Nadia T. Kaltcheva, Kirsten Kraiberg Knudsen, Soeren Schack Larsen, Cédric Ledoux, Raslan Leguet, Cécile Loup, Jean Mouette, David Mouillet, Anton Norup Soerensen and Lambertus Leonardus Vos.

On the other hand, we would like to thank Bernard Nicolet, Noël Cramer and Gilbert Burki, from Geneva Observatory, who provided us with many photometric data on the Geneva system.

Last, but not least, we are also grateful to Paula Sartoretti who gave us very useful help during the 1996-1997 campaign, and J. Bouvier for his very fruitful comments on the paper.

\section{References}

Aumann H.H., Gillett F.C., Beichman C.A., et al., 1984, ApJ 278, L23

Backman D.E., Paresce F., 1993, in Protostars and Planets III, Levy E.H., Lunine J.I., Matthews M.S. (eds.). Tucson: Univ. Arizona Press

Beust H., Vidal-Madjar A., Ferlet R., Lagrange-Henri A.M., 1991, A\&A 241, 488

Beust H., Lagrange-Henri A.M., Crawford I.A., et al., 1998, A\&A 338, 1015

Crawford D.L., Barnes J.V., 1970, AJ 75, 978

Ferlet R., Hobbs L.M., Vidal-Madjar A., 1987, A\&A 185, 267

Florentin Nielsen R., 1993, IAU Coll. 136, 213

Florentin Nielsen R., Norrengaard P., Olsen E.H., 1987, ESO Messenger 50, 45

Hoffleit D., Warren Jr. W.H., 1991, The Bright Star Catalog, 5th Revised Edition

Lagage P.O., Pantin E., 1994, Nat 369, 628

Lamers H.J.G.L.M., Lecavelier des Etangs A., Vidal-Madjar A., 1997, A\&A 328, 311

Lecavelier des Etangs A., Deleuil M., Vidal-Madjar A., et al., 1995a, A\&A 299, 557

Lecavelier des Etangs A., Deleuil M., Vidal-Madjar A., et al., 1995b, in Circumstellar Dust Disk and Planet Formation, Ferlet R., Vidal-Madjar A. (eds.). Éditions Frontières

Lecavelier des Etangs A., Vidal-Madjar A., Ferlet R., 1996, A\&A 307, 542

Lecavelier des Etangs A., Vidal-Madjar A., Burki G., et al., 1997, A\&A 328, 311

Mouillet D., Lagrange A.M., Beuzit J.L., Renaud N., 1997, A\&A 324, 1083

Olsen E.H., 1994, A\&AS 106, 257

Soderblom D.R., Duncan D.K., Johnson D.R.H., 1991, ApJ 375,722

Stepień K., Geyer E., 1995, Acta Astron. 45, 661

Vidal-Madjar A., Ferlet R., Lecavelier des Etangs A., et al., 1995, Astrophys. Space Sci. 223, 198

Vidal-Madjar A., Lecavelier des Etangs A., Ferlet R., 1998, Planet. Space Sci. 46, 629

Waters L.B.F.M., Van Den Ancker M.E., Baas F., et al., 1995, A\&A 299, 173 